\title{
Metodologias ativas de aprendizagem sobre anomalia craniofacial: o agente comunitário de saúde como formador de saberes
}

\author{
Taise Ferreira de Lima Galdino \\ Fonoaudióloga residente em Saúde da Família pelo Instituto de Medicina Integral Prof. Fernando Figueira \\ \taiselima95@gmail.com \\ Liliane Elise Souza Neves \\ Fonoaudióloga do Instituto de Medicina Integral Prof. Fernando Figueira, \\ Mestranda em Educação em Ciências: química da vida e saúde- UFRGS \\ $\triangle$ lilianeneves21@gmail.com \\ Amanda Almeida de Oliveira \\ Fonoaudióloga do Instituto de Medicina Integral Prof. Fernando Figueira, \\ Mestre em Saúde Materno Infantil e Doutora em Educação em Ciências: química da vida e saúde- UFRGS \\ $\bowtie$ mandafono@hotmail.com
}

\begin{abstract}
Resumo:
O Agente Comunitário de Saúde (ACS) constituindo um vínculo com a comunidade e o serviço de saúde, com isso faz necessário constantes atualizações sobre novas temáticas para atuarem na promoção e prevenção de saúde, sendo uma delas a anomalia craniofacial. Objetivo: Verificar a efetividade de um programa de aprimoramento com ACS utilizando metodologias ativas sobre anomalia craniofacial. Método: Trata-se de um relato de experiência de aprimoramento na temática de anomalia craniofacial, com pré e pós teste em grupo único. $\mathrm{O}$ aprimoramento foi realizado em dois encontros utilizando a metodologia ativa como, Metodologia da Problematização e TBL e um material virtual disponibilizado pelo Projeto Crânio-face Brasil de Bauru/SP. Os temas abordados foram: conceito sobre anomalia craniofacial, as causas, condutas de prevenção e tratamento. Para analisar o aproveitamento do aprimoramento pelos participantes foi aplicado um questionário pré e pós com perguntas sobre os assuntos abordados no decorrer do estudo. Resultados: A casuística constou de 27 ACS de Recife/PE correspondente as Unidades Básica de Saúde participantes vinculadas ao Hospital do IMIP. Os cursistas apresentaram um alto percentual de ocorrência de erros no questionário aplicado previamente ao aprimoramento em todo o grupo, sendo notório uma diminuição pós. Conclusões: Os resultados deste estudo comprovam êxito no aprimoramento com utilização de metodologias ativas proposto para os ACS sobre a temática da anomalia craniofacial.

Palavras-chave: Agentes Comunitários de Saúde, Anomalia craniofacial, Aprimoramento, Educação em Saúde.

\section{Active learning methodologies on craniofacial anomaly: the community health agent as a trainer of knowledge}

\begin{abstract}
:
The Community Health Agent ( $\mathrm{CHA}$ ) constituting a link with the community and the health service, therefore, it is necessary to constantly update on new topics to act in the promotion and prevention of health, one of them being the craniofacial anomaly. To verify the effectiveness of an improvement program with ACS using active methodologies on craniofacial anomaly. This is a report of an experience of improvement in the theme of craniofacial anomaly, with pre and post test in a single group. The improvement was carried out in two meetings using the active methodology, such as Problem Methodology and TBL and a virtual material made available by the Crânio-face Brasil Project
\end{abstract}


in Bauru / SP. The topics covered were: concept of craniofacial anomaly, causes, prevention and treatment. To analyze the improvement of the participants' improvement, a pre and post questionnaire was applied with questions on the subjects covered during the study. The sample consisted of 27 CHA from Recife / PE corresponding to the participating Basic Health Units linked to the IMIP Hospital. The course participants showed a high percentage of occurrence of errors in the questionnaire applied prior to the improvement in the whole group, with a notable post decrease. The results of this study prove successful in the improvement with the use of active methodologies proposed for the CHA on the theme of craniofacial anomaly.

Keywords: Community Health Agents, Craniofacial anomaly, Enhancement, Health education.

\title{
Metodologías de aprendizaje activo sobre anomalías craneofaciales: el agente de salud comunitario como formador de conocimiento
}

\begin{abstract}
Resumen:
El Agente de Salud de la Comunidad (CHA) constituye un vínculo con la comunidad y el servicio de salud, por lo tanto, es necesario actualizar constantemente los nuevos temas para actuar en la promoción y prevención de la salud, siendo uno de ellos la anomalía craneofacial. Verificar la efectividad de un programa de mejora con SCA utilizando metodologías activas en anomalías craneofaciales. Este es un informe de una experiencia de mejora en el tema de anomalía craneofacial, con prueba previa y posterior en un solo grupo. La mejora se llevó a cabo en dos reuniones utilizando la metodología activa, como la Metodología del problema y TBL y un material virtual puesto a disposición por el Proyecto Crânio-face Brasil en Bauru / SP. Los temas tratados fueron: concepto de anomalía craneofacial, causas, prevención y tratamiento. Para analizar la mejora de la mejora por parte de los participantes, se aplicó un cuestionario previo y posterior con preguntas sobre los temas cubiertos durante el estudio. La muestra consistió en 27 CHA de Recife / PE correspondientes a las Unidades Básicas de Salud participantes vinculadas al Hospital IMIP. Los participantes del curso tuvieron un alto porcentaje de errores que ocurrieron en el cuestionario aplicado antes de la mejora en todo el grupo, con una notable disminución posterior. Los resultados de este estudio tienen éxito en la mejora con el uso de metodologías activas propuestas para el CHA en el tema de anomalía craneofacial.
\end{abstract}

Palabras clave: Agentes Comunitarios de Salud, Anomalía Craneofacial, Mejora, Educación Sanitaria.

\section{INTRODUÇÃO}

O Ministério da Saúde criou em 1994 o Programa Saúde da Família (PSF) como uma estratégia de contribuir para a reorganização da atenção básica em vista dos resultados positivos obtidos pelo Programa de Agentes Comunitários de Saúde (PINTO, GIOVANELLA, 2018; BRASIL, 2017).

O Agente Comunitário de Saúde (ACS) foi inserido como um profissional fundamental na Estratégia da Saúde da Família (ESF) uma vez que o mesmo possui papel ímpar, pois diferentemente de todos os outros profissionais de saúde, ele está inserido na realidade da comunidade em que atua conhecendo as demandas e atuando como uma fundamental peça nas ações de promoção e prevenção de agravos à saúde (PERUZZO et al., 2018; PAULA et al., 2017). 
Constantes atualizações são necessárias sobre novas temáticas para atuação desses profissionais na promoção e prevenção de saúde abrangendo as necessidades da comunidade e os qualificando para boas práticas de saúde (NEVES et al., 2018; SANTOS et al., 2011). o programa de educação permanente em saúde pode ser uma estratégia para esses profissionais aprimorarem nas temáticas atuais, entre elas, anomalia craniofacial (NEVES, 2017; ARAÚJO, et al., 2015).

A anomalia craniofacial é considerada um dos defeitos congênitos humanos mais frequentes, causado pelo crescimento e/ou desenvolvimento anormal das estruturas das partes moles da cabeça e face e/ou ossos (BOYADJIEV, 2018; NEVES, et al., 2018; SOUSA et al., 2017).

A prevalência global revela 8 milhões dos nascimentos com defeitos congênitos graves ocorrem nos países em desenvolvimento (OPAS, 2020). Segundo a Organização Mundial de Saúde (OMS), a anomalia craniofacial é reconhecida como importante problema de saúde pública, devido ao não tratamento ou do tratamento ineficiente (GARIB et al., 2010; SOUSA et al., 2017).

Em virtude da alta porcentagem, cerca de 70\% dos defeitos congênitos podem ser prevenidos ou ter seu impacto minimizado em termos de mortalidade precoce, por meio de melhorias na qualidade da atenção à saúde (MDBDF, 2006; MONLLÉO, 2008).

Diante deste cenário, o ACS apropriado do conhecimento sobre à anomalia craniofacial pode ser um facilitador na prevenção, na identificação, encaminhamentos e gerenciamento da comunidade (COSTA et al., 2016). Para isso, faz-se necessário a elaboração de estratégias de educação que utilizem metodologias facilitadoras, contribuindo em um processo de aprendizagem ativa, e fortalecendo os saberes pré existentes destes sujeitos, neste campo o uso de metodologias ativas se apresentam como uma ferramenta eficaz.

Embora sejam várias as metodologias e técnicas educacionais construtivistas, este estudo propôs se concentrar em dois modelos, sendo a metodologia da problematização e o Team-Based Learning- TBL (BERBEL, 1999; OLIVEIRA et al., 2018).

A Metodologia da Problematização é uma proposta de ensino-aprendizagem por considerar como premissa da educação a realidade circundante ao indivíduo, suas vivências 
e experiências, seus saberes e conhecimentos apriorísticos sendo realizado uma troca de conhecimentos entre os participantes. Assim, numa relação dialógica e educativa, direciona os saberes para uma convergência: a mudança individual e coletiva e, consequentemente, a transformação da realidade de maneira crítica e criativa (BERBEL, 1999).

O Team-Based Learning- TBL é uma estratégia educacional constituída por um conjunto de práticas dividida em três etapas. Ela visa promover o desenvolvimento de equipes de aprendizagem de alto desempenho e fornecer a estas equipes oportunidades para se envolver em tarefas de aprendizagem significativas (KRUG et al., 2016).

Neste modelo, a primeira etapa é o preparo, a qual é realizado um preparo prévio do estudante para uma tarefa proposta pelo professor fora da sala de aula. A segunda é a garantia do preparo, em que consiste num teste individual e após em equipe, com feedback, sendo ambos realizado em sala de aula. Por fim, a aplicação dos conceitos, envolve a execução de várias tarefas em equipe propostas pelo professor, que, geralmente, envolvem resolução de problemas e tomadas de decisão, seguidas por uma apresentação e feedback. Todos os processos que ocorrem nestas fases são avaliados (KRUG et al., 2016; OLIVEIRA et al., 2018).

Nesse contexto, esta pesquisa teve por objetivo verificar a efetividade de um programa de aprimoramento com ACS utilizando metodologias ativas sobre anomalia craniofacial.

\section{METODOLOGIA}

Trata-se de um relato de experiência realizado nas Unidades Básica de Saúde (UBS) vinculadas ao Instituto de Medicina Integral Prof. Fernando Figueira (IMIP) no período de outubro a dezembro de 2019.

O estudo foi aprovado pelo Comitê de Ética em Pesquisa do IMIP número 46848415.0.0000.5201.

Os ACS foram submetidos a um aprimoramento na temática da anomalia craniofacial, estes foram divididos em três grupos, no período do estudo foram realizados dois encontros 
presenciais totalizando oito horas de atividades, sendo executada nas ESF correspondente aos ACS.

O primeiro encontro teve como proposta a aplicação de um questionário com dezoito perguntas objetivas elaboradas pelas pesquisadoras com intuito de verificar o nível de conhecimento prévio (Quadro 1, página seguinte), em seguida, foram expostos os conteúdos essenciais para um desejável aprimoramento: “o que é anomalia craniofacial?" e "quais são suas causas" baseada na Metodologia da Problematização (BERBEL, 1999).

No período entre o primeiro e o segundo encontro foi disponibilizado por uma rede social, WhatsApp, um material virtual "Manual de cuidados de saúde e alimentação da criança com fenda oral" do projeto Crânio- Face Brasil (MONLLEÓ, MENDES, GIL-DA-SILVA-LOPES, 2014) com o intuito de ser um ambiente de trocas de experiências, aprendizado e um elo entre os mediadores e os participantes do estudo.

No segundo encontro, foi utilizado o Team-Based Learning- TBL (OLIVEIRA et al., 2018), metodologia escolhida para atingir os objetivos de aprendizagem no aprimoramento, sendo expostos os conteúdos de condutas práticas: “condutas de prevenção" e "tratamento". Ao final do aprimoramento, foi um contra prova do mesmo questionário (Quadro 1). 
Quadro 1- Questionário aplicado previamente e após o aprimoramento

\begin{tabular}{|c|c|c|}
\hline \multicolumn{3}{|l|}{$\begin{array}{l}\text { Nome: } \\
\text { Idade:__-_-_ Sexo: _---- Grau de Escolaridade: } \\
\text { Tempo de Serviço como ACS: }\end{array}$} \\
\hline Questionamentos & Sim & Não \\
\hline 2. Sei o que significa anomalias craniofaciais? & & \\
\hline 3. Conheço as principais origens das anomalias craniofaciais? & & \\
\hline 4. Sei identificar ao menos uma das mais comuns anomalias craniofaciais? & & \\
\hline 5. Acredito que não existe uma causa única para a formação das anomalias craniofaciais? & & \\
\hline 6. Conheço as principais causas das anomalias craniofaciais? & & \\
\hline 7. A hereditariedade e a genética são as únicas causas das anomalias craniofaciais? & & \\
\hline $\begin{array}{l}\text { 8. Consigo realizar atividades, como palestras, grupos, salas de espera e visitas } \\
\text { domiciliares, relacionadas às anomalias craniofaciais? }\end{array}$ & & \\
\hline $\begin{array}{l}\text { 9. Sinto-me preparado (a) para visitar um usuário em minha micro área de abrangência } \\
\text { que tenha anomalias craniofacial? }\end{array}$ & & \\
\hline 10. Sei dar orientações às famílias e usuários que apresentem anomalias craniofaciais? & & \\
\hline $\begin{array}{l}11 \text { Acho que o principal responsável pelas orientações aos familiares com crianças que } \\
\text { apresentem anomalias craniofaciais deve ser dever do: } \\
\text { ( ) Médico da família; ( ) Enfermeiro; ( ) Técnico em Enfermagem; ( ) ACS; ( ) De } \\
\text { todos citado acima }\end{array}$ & & \\
\hline 12. Conheço as condutas de prevenção para as anomalias craniofaciais? & & \\
\hline $\begin{array}{l}\text { 13. Sinto-me seguro para discutir com minha equipe o melhor direcionamento para o } \\
\text { desenvolvimento da criança? }\end{array}$ & & \\
\hline $\begin{array}{l}\text { 14. Sei explicar uma mãe sobre a melhor forma de alimentar um bebê com anomalias } \\
\text { craniofacial? }\end{array}$ & & \\
\hline $\begin{array}{l}\text { 15. Acredito que um bebê, mesmo com anomalias craniofacial, pode ser alimentado em } \\
\text { qualquer posição? }\end{array}$ & & \\
\hline $\begin{array}{l}\text { 16. Conheço as estratégias de aplicação dos meios de prevenção das anomalias } \\
\text { craniofaciais? }\end{array}$ & & \\
\hline $\begin{array}{l}\text { 17. Acho necessário que uma criança ou adulto que possua anomalia craniofacial deva } \\
\text { ser encaminhado a um centro de referência? }\end{array}$ & & \\
\hline $\begin{array}{l}\text { 18. Conheço o cetro de referência para as anomalias craniofaciais do estado de } \\
\text { Pernambuco? }\end{array}$ & & \\
\hline
\end{tabular}

Fonte: Própria 


\section{RESULTADOS}

Observa-se, na Figura 1, alto percentual de ocorrência de erros no questionário aplicado previamente ao aprimoramento em todo o grupo, sendo notório pós aprimoramento, uma diminuição de ocorrência de erros nas respostas dos sujeitos pesquisados.

Ressalta-se que apenas 4 sujeitos (14,8\%) apresentou um percentual de mais de $70 \%$ de acertos no questionário prévio ao aprimoramento. Enquanto no pós, notado que os 27 sujeitos (100\%) apresentaram um percentual maior de 70\% no mesmo questionário aplicado posteriormente ao aprimoramento.

Figura 1. Pares de respostas corretas no período pré $\mathrm{x}$ pós treinamento por participante

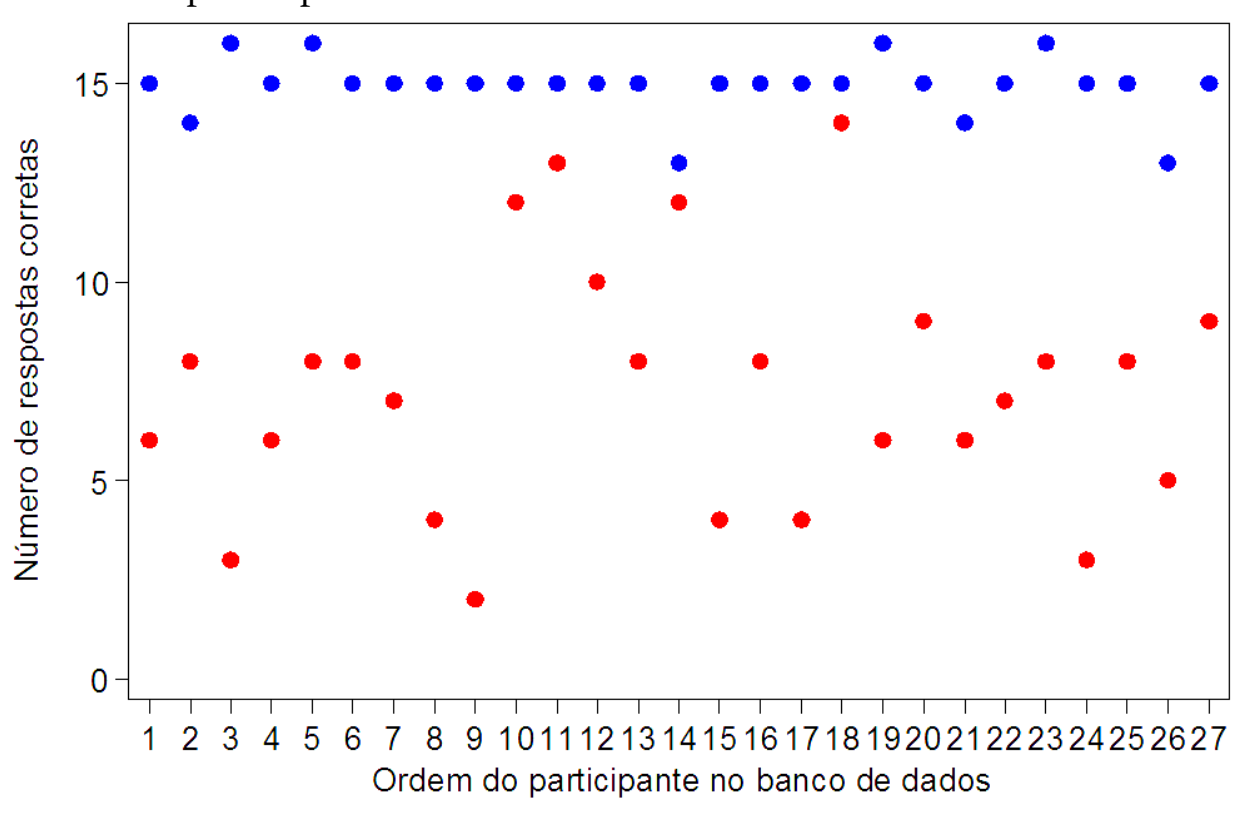

- No. de acertos pós - No. de acertos pré

Fonte: Própria

O resultado da Tabela 1 mostra que a média de acerto no pós aprimoramento foi praticamente o dobro daquela obtida no pré aprimoramento. 0 aumento de 7,6 pontos foi estatisticamente significante de acordo com teste $t$ de Student para amostras pareadas $(t=$ $12,0 ; \mathrm{gl}=26 ; \mathrm{p}<0.001)$. 
Tabela 1- Estatísticas descritivas do número de respostas corretas obtidas no pré e pós aprimoramento.

\begin{tabular}{lcccl}
\hline VARIÁVEL & N & MÉDIA \pm DP & MÍNIMO & MÁXIMO \\
\hline Pré & 27 & $7,3 \pm 3,1$ & 2 & 14 \\
Pós & 27 & $14,9 \pm 0,7$ & 13 & 16 \\
Diferença = Pós -Pré & 27 & $7,6 \pm 3,3$ & 1 & 13 \\
\hline
\end{tabular}

Fonte: Própria

Quando comparados os sujeitos de estudo por número de erros nas alternativas representadas na Figura 2, foi verificado um alto percentual para alternativas dos seguintes números: $6,8,12,13,14,15,16,18$. Enquanto, no período pós formação, pode ser notado uma redução de número de erros por alternativas, sendo observado uma conservação de erros nas alternativas dos números 7,8,14,15 e 18. No entanto, apresentadas com um baixo percentual de 3 (11\%) das pessoas mantem o erro nestas alternativas, exceto pela alternativa número 15 com 19 (70\%) dos sujeitos ainda permanecem com conceitos irreais.

Figura 2. Número de erros no pré aprimoramento

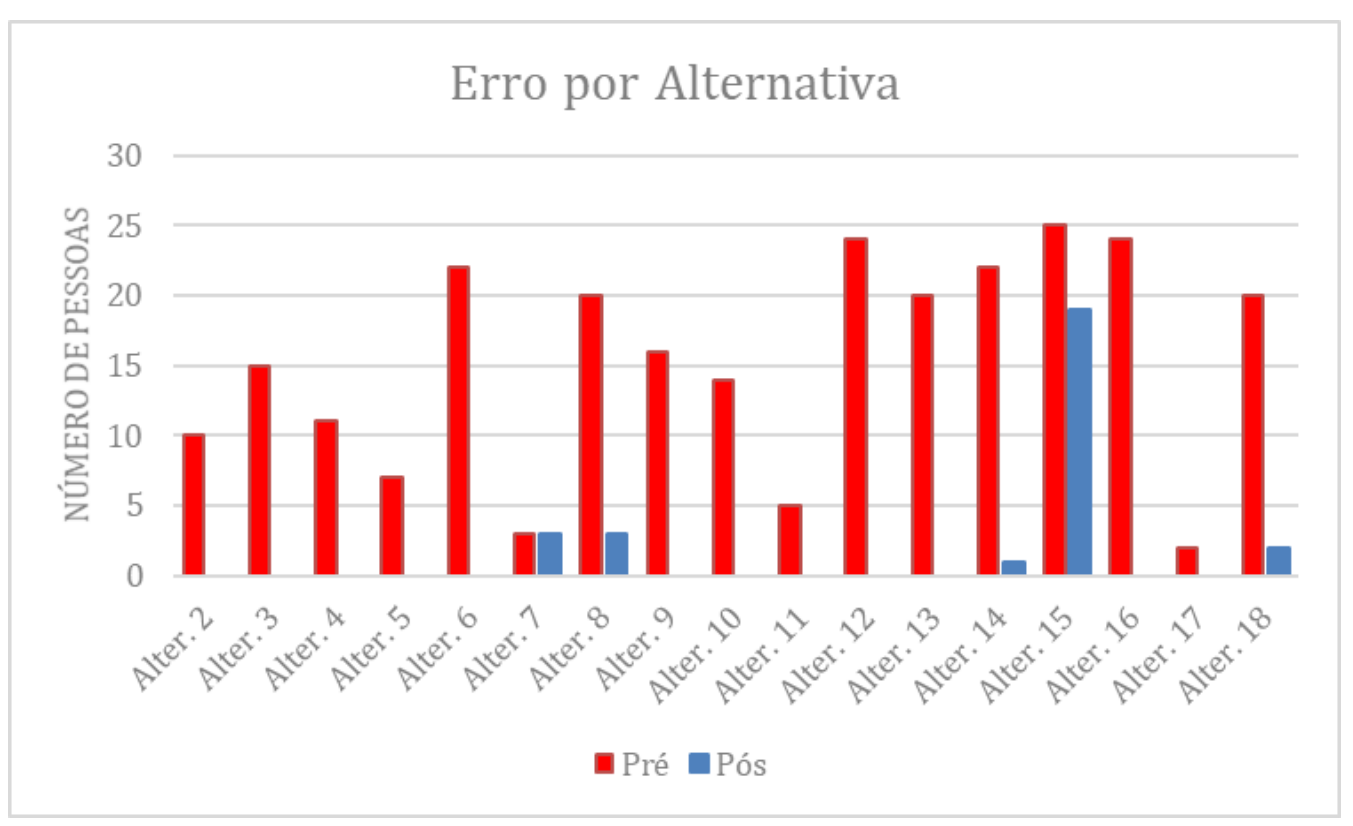

Fonte: Própria

O uso do TBL durante o aprimoramento representa na figura abaixo com o percentual de acerto para atividade aplicada individualmente e em grupo. É possível notar em ambos os 
momentos houve um elevado percentual de acertos, sendo considera quase $100 \%$ durante a atividade em grupo, e não menos importante, o individual mostrou- se com quase $90 \%$ de resultado positivo.

Figura 3. Resultado TBL Individual X Grupo.

\section{Resultado TBL Individual X Grupo}
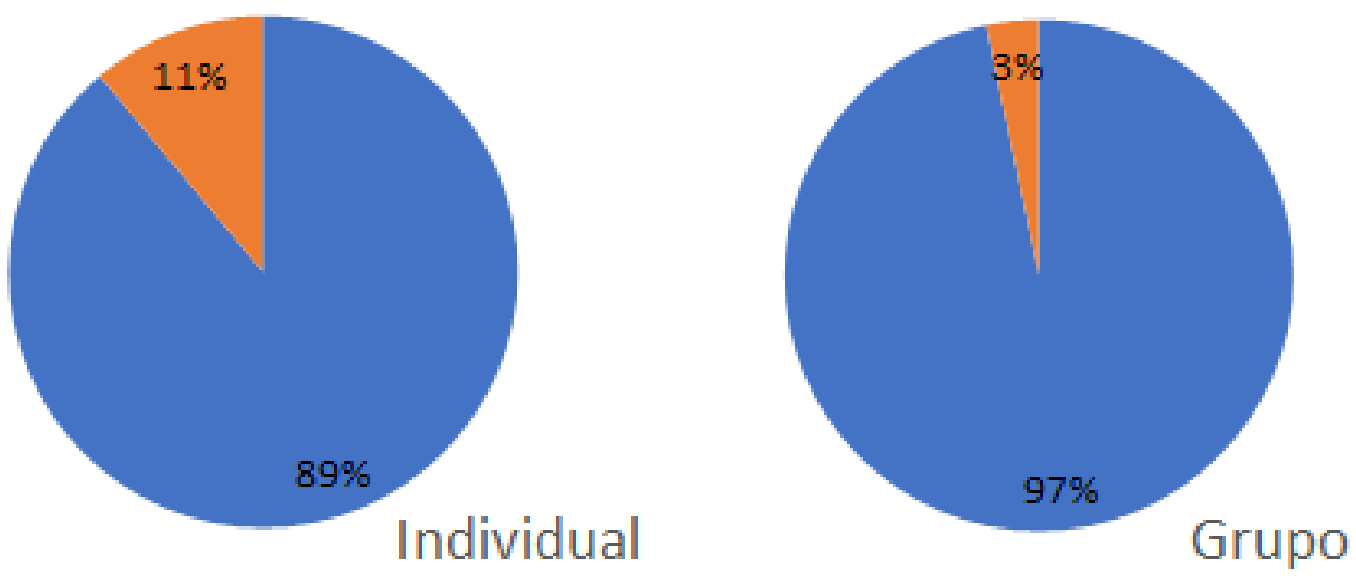

Respostas Corretas nespostas Erradas

Fonte: Própria

\section{DISCUSSÃO}

A aplicação da educação em saúde tem sido de formas distintas, partindo do princípio das necessidades e ferramentas envolvidas para determinados grupos. A vinculação com a unidade de saúde, bem como a expansão das informações colabora positivamente no acesso e/ou melhoria das condições de saúde em diversos segmentos (NASCIMENTO et. al., 2017; DIAS et. al., 2015).

Cada vez mais a educação em saúde mediada por recursos de mídia é consolidada como prática eficaz para o aprimoramento de indivíduos que possuem informações superficiais sobre determinados conceitos. Assim, à tecnologia da informação constitui uma oportunidade para reduzir barreiras geográfica e a extensão do processo de atenção praticada 
nos grandes centros onde a serviços especializados (NASCIMENTO et al., 2017; GODOY, GUIMARAES, ASSIS, 2014).

Tendo em vista que ACS é responsável no acompanhamento das famílias de uma determinada área e possui papel específico dentro da unidade de saúde, o aprimoramento visou abarcar a temática sobre a anomalia craniofacial de modo didático, simples e com uso de metodologias ativas afim de, aproximar esses profissionais sobre uma temática não tão discutida em seus cadernos de formação (NEVES et. al., 2018; BRASIL, 2017; BRASIL, 2009).

Com isso, antes de iniciar o aprimoramento, foi aplicado um questionário para medição do nível de conhecimento de cada ACS acerca da anomalia craniofacial. Dentro dos dados coletados, foi possível observar um número acentuado de dúvidas e conceitos não reais sobre a anomalia craniofacial representada na Figura 1.

No entanto, verificasse que esses profissionais não obtiveram uma qualificação que o deixasse apto para ter uma habilidade que correspondesse às demandas que lhes são apresentadas diariamente, sendo uma delas, a anomalia craniofacial (NEVES et. al., 2018; BRASIL, 2009).

Ao observar a Figura 2, houve um alto percentual de erros na aplicação do questionário prévio ao aprimoramento concordam com achados da literatura em que os ACS apresentam um conhecimento reduzido sobre a anomalia craniofacial. Em vista que, muitos de seus saberes técnico-científicos são advindos das vivências em equipe e saberes populares embebidos da cultura local (MACIAZEKI-GOMES et al., 2016; PERUZZO et al., 2018).

De tal modo, o uso de recursos de mídia e educação permanente na estratégia de educação em saúde é capaz de construir as concepções práticas e ainda, permiti desenvolver a capacidade crítica dos profissionais (CORREA, 2012; NASCIMENTO et. al., 2017).

Assim, o estudo se baseou no uso de dois modelos de metodologia ativa com intuito de abranger de forma dinâmica toda a temática levantada. Para isso, foi construído materiais didáticos e acessíveis para exposição do tema com uso de recursos digitais de animação, como: figura, quebra-cabeça, material interativo, animações e ilustrações passam a informação de forma positiva alcançando a simpatia dos participantes, facilitando sua aprendizagem. 
As estratégias de metodologias ativas foram divididas por módulos, sendo o uso da Metodologia da Problematização, no primeiro modulo do aprimoramento. Nesta fase, constituiu na proposta de apresentar conteúdo por meio da observação da realidade de modo crítico fazendo relacionar com a realidade da temática trabalhada. Com isso, trata-se numa aprendizagem a partir dos conhecimentos prévios favorecendo contato e comparação com a realidade e assim, pós reflexão da problematização seja possível chegar numa hipótese de solução para a temática estudada (RIBEIRO et al., 2016; BERBEL, 1999).

No segundo modulo, foi utilizada a metodologia, Team-Based Learning- TBL na concepção, no processo de aprendizagem e na avaliação da formação dos ACS. Como proposto na metodologia deste estudo, no intervalo entre os módulos, foi disponibilizado o material virtual "Manual de cuidados de saúde e alimentação da criança com fenda oral" do projeto CrânioFace Brasil para que no retorno do segundo modulo os participantes pudessem realizar a dinâmica proposta pela metodologia (OLIVEIRA et al., 2018; MONLLEÓ, MENDES, GIL-DASILVA-LOPES, 2014).

O referido TBL, em sua fase de garantia do preparo é dividido em dois momentos, aplicação de um questionário individual com dez alternativas, na sequência, formado pequenos grupos para concessão de uma única resposta assertiva para alterativas respondidas individualmente, e por fim, cada grupo apresenta para os demais a conclusão das respostas escolhidas e justificadas para concessão de pontual grupal e individual para aquelas corretas (OLIVEIRA et al., 2018).

Com isso, essa aplicação forneceu boa resposta para os participantes deste estudo, sendo possível notar na Figura 3, um alto percentual de acertos na etapa individual com 240 (89\%) respostas assertivas de um total de 270 (100\%), como também em grupo, 5 (97\%) apresentaram $100 \%$ de acerto, enquanto 1 grupo (3\%) acertou 8 questões (80\%) da atividade.

Diante desses dados, é possível verificar que durante a atividade em grupo ocorre um melhor percentual, sendo já comprovado na literatura que atividades dinâmicas grupais fazem com que os participantes tenha uma melhor troca de seus conhecimentos, em organizar os conceitos e é possível verificar efetividade na forma mais articulada quanto aos conhecimentos necessários para responder às demandas e necessidade de saúde estudada e 
auxiliar na transformação da realidade das condições de vida e saúde locais (OLIVEIRA et al., 2018; COLARES, OLIVEIRA, 2018).

Considerando abordagem metodológica ativa, os dados antes do processo de aprimoramento através da estratégia de educação, apenas 199 (43,35\%) das respostas obtidas pela população pesquisada refletiam o conhecimento prévio quanto a temática exposta. A maior parte das respostas obtidas antes do processo de metodologia de trabalho, 260 (56,65\%) reflete um desconhecimento geral. Ao fim da estratégia, houve uma mudança no padrão das respostas. De 199 (43,35\%) para 431 (93,8\%) em respostas assertivas que refletem um aumento no nível de conhecimento, e em comparação as respostas que refletem desconhecimento a queda foi de $260(56,65 \%)$ para $28(6,1 \%)$, sendo este aumento estatisticamente significante (Teste de McNemar: $\mathrm{p}<0.001$.) (GARCIA, BAPTISTA, 2014).

Este aumento pós aprimoramento predispõe a validade do uso da estratégia de educação em saúde utilizada por meio da ferramenta de tele-educação como viável e positiva ao aprimoramento de ACS (ARAÚJO et al., 2015; ALVARENGA et al., 2008). Em vista da considerável redução de erros observado pós aprimoramento, Figura 2, relevando um aumento expressivo sobre o nível de conhecimento dos pesquisados quanto à temática da anomalia craniofacial, pode-se afirmar que, a estratégia é efetiva resultando num feedback positivo na compreensão da temática.

Retomando os dados já observados na Figura 2 com seu alto índice de erros nas alternativas respondidas previamente ao aprimoramento e que pós aprimoramento, foi notado uma redução dos erros. Esses dados do estudo corrobora com os dados coletados na literatura, em que foi observado efetividade da absorção de informações voltadas a área de atuação da fonoaudiologia e o uso metodologias ativas de ensino em saúde (ALVARENGA et al., 2008; NEVES, 2017; NEVES et al., 2018).

Ainda assim, mesmo após o aprimoramento, um percentual elevado para a alternativa quinze com 19 (70\%) dos sujeitos ainda permanecem com conceitos errôneo sobre postura na alimentação da criança com anomalia craniofacial. Cabe as hipóteses, "o conceito passado não foi suficiente para suprir essa demanda", "os participantes generalizaram a postura alimentar apenas para a anomalia mais comum que é usada na fissura labiopalatina". 
No que diz respeito aos dados obtidos através da média do questionário aplicado previamente e após (Tabela 1), descreve que o aprimoramento em anomalia craniofacial teve boa significância, mostrando uma diferencia dos dois momentos de 7,6 (média) reafirmando que o uso dessas metodologias, pode ser notado benefícios nas aptidões adquiridas pelos ACS durante o processo de aprimoramento em: troca de informação/ conhecimentos, habilidades de comunicação e argumentação, atenção dos conceitos apresentados durante o momento presencial no aprimoramento e melhoria da interdependência positiva (OLIVEIRA et al., 2018; CORREA, 2012; NASCIMENTO et. al., 2017).

Por tratar-se de um processo de aprimoramento em saúde dinâmico, o uso dessas metodologias proporcionou o sujeito como ator deste aprendizado, uma melhor absorção do conteúdo abordado e favorecendo uma prática eficaz.

As práticas de educação em saúde oferecem benefícios que estabelecem um importante alternativa para melhorar o acesso dos trabalhadores do Sistema Único de Saúde (SUS) à educação permanente, por permitir ao profissional a exercício de discussões teóricas reflexivas, baseadas nas necessidades da população envolvida e estimulando a expansão do conhecimento aprendido para um número maior de pessoas (DINIZ et. al., 2016).

No entanto, o desenho de novas pesquisas envolvendo aprimoramento para o ACS relacionada as demandas da comunidade, deve incluir uma escala longitudinal para verificar se é efetivo na problematização da área vivenciada. Em vista que os ACS terem participado de dos módulos, durante duas semanas, não foi possível ser realizado um controle rigoroso das atividades que o ACS desenvolveu nos pós aprimoramento, o que permitiria analisar se todos tiveram ou não a mesma oportunidade de aplicação do conteúdo, o que envolve a abrangência da área de atuação, número de famílias visitadas, contato com crianças com anomalia craniofacial, dentre outros.

Assim, ressalta-se a importância de desenvolvimento de estudos futuros que realizem um controle das atividades desempenhadas pelos profissionais pós-aprimoramento.

Portanto, são necessárias novas análises e investigações científicas incluindo esse modelo de educação em saúde e utilização dessa ferramenta com outros profissionais de saúde sobre a temática para verificar eventuais benefícios na prática do atendimento na atenção básica. 


\section{CONCLUSÃO}

Os resultados deste estudo comprovam êxito do programa de aprimoramento com utilização de metodologias ativas proposto para os ACS sobre a temática da anomalia craniofacial. Deste modo, pode-se inferir que o aprimoramento oferecido permitiu o aumento do conhecimento dos ACS quanto o conteúdo abordado, sendo considerada uma ferramenta válida e aceita pelos participantes, logo porque, fomentou a discussão do conteúdo de forma dinâmica propiciando o conhecimento necessário sobre o conteúdo abordado.

Há necessidade da educação permanente para esses profissionais nas diferentes áreas da saúde, fazendo com que aumentem seu repertório de informações, as quais sejam capazes de promover a saúde, uma vez que o ACS é um ator que desempenha um papel fundamental no Sistema Único de Saúde.

\section{REFERÊNCIAS}

ALVARENGA, K. F.; BEVILACQUA, M. C.; MARTINEZ, M. A. N. S.; MELO, T. M.; BLASCA, W. Q.; TAGA, M. F. L.. Proposta para capacitação de agentes comunitários de saúde em saúde auditiva. Pró-fono Revista de Atualização Científica, v. 20, n. 3, p. 171-176, 2008. http://dx.doi.org/10.1590/s0104-56872008000300006.

ARAÚJO, E. S.; JACOB-CORTELETTI, L. C. B.; ABRAMIDES, D. V. M.; ALVARENGA, K. F.. Capacitação de agentes comunitários de saúde na área de saúde auditiva infantil: retenção da informação recebida. Revista Cefac, v. 17, n. 2, p. 445-453, 2015. http://dx.doi.org/10.1590/1982-0216201511913.

BERBEL, N. A. N.. Metodologia da Problematização e os ensinamentos de Paulo Freire: uma relação mais que perfeita. In: BERBEL, N. A (org.). Metodologia da problematização - fundamentos e aplicações. Londrina: Ed. UEL, 1999.

BRASIL. Ministério da Saúde. Portaria nº 2.436, de 21 de setembro de 2017. Aprova a Política Nacional de Atenção Básica, estabelecendo a revisão de diretrizes para a organização da Atenção Básica, no âmbito do Sistema Único de Saúde (SUS). Brasília, DF: $\quad$ Ministério da $\quad$ Saúde, $2017 . \quad$ Disponível em: <https://bvsms.saude.gov.br/bvs/saudelegis/gm/2017/prt2436_22_09_2017.html>. Acesso em: 30 nov. 2019.

BRASIL. Ministério da Saúde. O trabalho do Agente Comunitário de Saúde. Brasília, DF: Ministério da Saúde, 2009 a. Disponível em: <http://189.28.128.100/dab/docs/publicacoes/geral/manual_acs.pdf>. Acesso em: 30 jan. 2020.

COLARES, K.T.P.; OLIVEIRA, W.. Metodologias Ativas na formação profissional em saúde: uma revisão. Revista Sustinere, v. 6, n. 2, p. 300-320, 2019. http://dx.doi.org/10.12957/sustinere.2018.36910.

COSTA, T.L.; SOUZA, O. M. V.; CARNEIRO, H. A.; NETTO, C. C.; PEGORARO-KROOK, M. I.; DUTKA, J. C. R.. Material multimídia para orientação dos cuidadores de bebês com fissura labiopalatina sobre velofaringe e palatoplastia primária. Codas, v. 28, n. 1, p. 10-16, 2016. http://dx.doi.org/10.1590/2317-1782/20162014126.

DIAS, R. S.; MARQUES, A. F. H.; DINIZ, P. R. B.; SILVA, T. A. B.; COFIEL, L.; MARIANI, M. M. C.; SALGADO, C. L.; OLIVEIRA, A. E. F.; MIGUEL FILHO, E. C.; WEN, C. L.. Telemental health in Brazil: past, present and integration into primary care. Archives of Clinical Psychiatry, v. 42, n. 2, p. 41-44, 2015. http://dx.doi.org/10.1590/0101-608300000000046.

DINIZ, P.R.B.; SALES, F. J. R.; NOVAES, M. A.. Providing Telehealth Services to a Public Primary Care Network: the experience of redenutes in pernambuco, brazil. Telemedicine And E-health, v. 22, n. 8, p. 694-698, 2016. http://dx.doi.org/10.1089/tmj.2015.0209.

GARCIA, R.M.; BAPTISTA, R.. Educação a distância para a qualificação dos profissionais do sus: perspectivas e desafios. Baiana Saúde Pública, v. 31, n. 1, p.70-78, 2007. 
Metodologias ativas de aprendizagem sobre anomalia craniofacial:

o agente comunitário de saúde como formador de saberes

GARIB, D. G.; Alencar, B. M.; Ferreira, F. V.; Ozawa, T. O.. Anomalias dentárias associadas: o ortodontista decodificando a genética que rege os distúrbios de desenvolvimento dentário. Dental Press J. Orthod., v. 15, n. 2, p.138-157, 2010.

GODOY, S.C.B.; GUIMARÃES, E. M. P.; ASSIS, D. S. S.. Evaluation of the training of nurses in basic health units through telenfermagem. Escola Anna Nery - Revista de Enfermagem, v. 18, n. 1, p. 148-155, 2014. http://dx.doi.org/10.5935/1414-8145.20140022.

KRUG, R.R.; VIEIRA, M. S. M.; MACIEL, M. V. A.; ERDMANN, T. R.; VIEIRA, F. C. F.; KOCH, M. C.; GROSSEMAN, S.. O "Bê-Á-Bá" da Aprendizagem Baseada em Equipe. Revista Brasileira de Educação Médica, v. 40, n. 4, p. 602-610, 2016. http://dx.doi.org/10.1590/1981-52712015v40n4e00452015.

MACIAZEKI-GOMES, R.C.; SOUZA, C. D.; BAGGIO, L.; WACHS, F.. O trabalho do agente comunitário de saúde na perspectiva da educação popular em saúde: possibilidades e desafios. Ciência \& Saúde Coletiva, v. 21, n. 5, p. 1637-1646, 2016. http://dx.doi.org/10.1590/1413-81232015215.17112015.

MARCH OF DIMES BIRTH DEFECTS FOUNDATION (MDBDF). March of dimes global report on birth defects: the hidden toll of dying and disabled children. (Org.). Christianson, A.; Howson C.P.; Modell, B. New York, p. 8-53, 2006. Disponível em: <https://www.marchofdimes.org/global-report-on-birth-defects-the-hidden-toll-of-dying-and-disabled-children-fullreport.pdf>. Acesso em: 2 nov. 2019.

MONLLEÓ, I.L.; MENDES, L.G.A.; GIL-DA-SILVA-LOPES, V.L. Manual de cuidados de saúde e alimentação da criança com fenda oral. (Org.). Projeto crânio-face Brasil. São Paulo, p. 1-23, 2014. Disponível em: <https://www.fcm.unicamp.br/fcm/sites/default/files/paganex/manual_fof_final.pdf $>$. Acesso em: 20 nov. 2019.

NASCIMENTO, C.M.B.; LIMA, M. L. L. T.; SOUSA, F. O. S.; NOVAES, M. A.; GALDINO, D. R.; SILVA, É. C. H.; LEITÃO, G. G. S.; SILVA, T. P. S.. Telefonoaudiologia como estratégia de educação permanente na atenção primária à saúde no Estado de Pernambuco. Revista Cefac, v. 19, n. 3, p. 371-380, 2017. http://dx.doi.org/10.1590/1982-0216201719314716.

NEVES, L.E.S.. Educational Strategy for Community Health Agents in the City of Recife: knowing Craniofacial Anomalies. Latin Am J Telehealth, v. 3, n. 4, p.256-260, 2017.

NEVES, L.E.S.; OLIVEIRA, A.A.; SILVA, BH.; MELO, D.B.; COUTO, J. M. L. A.; BARROS, N. C. G.; FIGUEIRA, M. A. S.; PEREIRA, R. M.. Use of media resources as educational strategy for the training of community health agents in craniofacial anomalies. Atin Am $\mathbf{J}$ Telehealth, v. 1, n. 5, p.28-32, 2018.

OLIVEIRA, B.L.C.A.; LIMA, S. F.; RODRIGUES, L. S.; PEREIRA JÚNIOR, G.A.. Team-Based Learning como Forma de Aprendizagem Colaborativa e Sala de Aula Invertida com Centralidade nos Estudantes no Processo Ensino-Aprendizagem. Revista Brasileira de Educação Médica, v. 42, n. 4, p. 86-95, 2018. http://dx.doi.org/10.1590/1981-52712015v42n4rb20180050.

ORGANIZAÇÃO PAN-AMERICANA DA SAÚDE (OPAS). Nascidos com defeitos congênitos: histórias de crianças, pais e profissionais de saúde que prestam cuidados ao longo da vida. Organização Pan-Americana da Saúde- Brasil, 2020. Disponível em: $\quad<$ https://www.paho.org/bra/index.php?option=com_content\&view=article\&id=6117:nascidos-com-defeitos-congenitoshistorias-de-criancas-pais-e-profissionais-de-saude-que-prestam-cuidados-ao-longo-da-vida\&Itemid=820>. Acesso em: 3 jan. 2020.

PAULA, M.C.; VIANNA, K. M. P.. Agravos fonoaudiológicos sob a ótica do agente comunitário de saúde. Revista Cefac, v. 19, n. 2, p. 221-232, 2017. http://dx.doi.org/10.1590/1982-021620171921016.

PERUZZO, H.E.; BEGA, A. G.; LOPES, A. P. A. T.; HADDAD, M. C. F. L.; PERES, A. M.; MARCON, S. S. The challenges of teamwork in the family health strategy. Escola Anna Nery, v. 22, n. 4, p.1-9, 2018. http://dx.doi.org/10.1590/2177-9465-ean-2017-0372.

PINTO, L.F.; GIOVANELLA, L.. Programa à Estratégia Saúde da Família: expansão do acesso e redução das internações por condiçõ es sensíveis à atenção básica (ICSAB). Ciência \& Saúde Coletiva, v. 6, n. 23, p.1903-1913, 2018.

SANTOS, K.T.; SALIBA, N. A.; MOIMAZ, S. A. S.; ARCIERI, R. M.; CARVALHO, M. L.. Agente comunitário de saúde: perfil adequado a realidade do Programa Saúde da Família? Ciência \& Saúde Coletiva, v. 16, n. 1, p.1023-1028, 2011. http://dx.doi.org/10.1590/s1413$\underline{81232011000700035}$

SOUSA, L.C.; MORAES, M. C. A. F.; SOUZA, C. D. R.; SILVA, H.; SILVA, É. G.; REIS, L. C. S.; SILVA, P. P.; MAXIMINO, L. P.. Ambiente virtual de aprendizagem: contribuições da terapia ocupacional a pais e familiares na assistência de crianças com anomalias craniofaciais. Cadernos Brasileiros de Terapia Ocupacional, v. 25, n. 2, p.255-266, 2017. http://dx.doi.org/10.4322/0104-4931.ctoA00926.

$(\mathrm{cc}) \mathrm{Br}$

Este trabalho está licenciado com uma Licença Creative Commons - Atribuição 4.0 Internacional. 